\title{
Simultaneous quantification of free and esterified cholesterol extracted from plasma by ${ }^{13} \mathrm{C}$ NMR spectroscopy
}

\author{
E. Baguet ${ }^{*}$, T. Magot ${ }^{2}$, K. Ouguerram ${ }^{2}$, R. J. Robins ${ }^{1}$ and R. J. Weesie ${ }^{1}$ \\ ${ }^{1}$ Laboratoire d'Analyse Isotopique et Électrochimique de Métabolismes, UPRESA CNRS 6006, \\ Université de Nantes, BP 92208, 2, rue de la Houssinière, 44322 Nantes Cedex 03, France \\ ${ }^{2}$ Centre de Recherche en Nutrition Humaine, groupe métabolisme, Hôtel Dieu, 1, place Ricordeau, \\ 44093 Nantes Cedex 01, France
}

\begin{abstract}
An efficient strategy for the simultaneous quantification of free and esterified cholesterol in a mixture by ${ }^{13} \mathrm{C}$ NMR spectroscopy is described. It is based on the choice as internal reference of cholesteryl bromide, which has a similar chemical structure and ${ }^{13} \mathrm{C}$ NMR spectral features to those of the analytes in the mixture. In this way, it is possible to obtain accurate results using a short recycle time and benefiting from the nuclear Overhauser enhancement. The analytical procedure has been used to quantify both free and esterified cholesterol in samples of known concentration. The measured values are in good agreement $\left(\mathrm{R}^{2}>0.998\right)$ with the expected values, thanks to the use of several resonance's within the target molecules, yielding a statistical average of the final concentration of each analyte. The method is shown to be suitable for the quantification of esterified and free cholesterol in a crude lipid mixture extracted from human blood.
\end{abstract}

Keywords. Quantitative ${ }^{13} \mathrm{C}$ NMR spectroscopy - cholesterol - cholesteryl ester.

$\begin{array}{ll}\text { Abbreviations used } \\ \text { Cr(AcAc) })_{3} & \text { triacetylacetonatochromium } \\ \text { FID } & \text { Free induction decay } \\ \eta & \text { nuclear Overhauser enhancement factor } \\ \text { LDL } & \text { Low density lipoprotein } \\ \text { NOE } & \text { nuclear Overhauser enhancement } \\ \text { TMS } & \text { Tetramethylsilane }\end{array}$

\section{Introduction}

Many different diseases are known to be associated with a disturbance in lipoprotein balance and composition. In order to understand the metabolic perturbations related to these diseases, it is important to determine precisely the concentrations of cholesterol and cholesteryl ester in plasma and in different classes of lipoproteins [1]. Classically, the different classes of lipoproteins are separated by ultracentrifugation and assessed by one of two methods. The first involves the measurement of the amount of cholesterol obtained after isolation of cholesterol and cholesteryl ester by chromatography and saponification of cholesteryl ester [1]. This involves long manipulations during which significant fractions of the analytes might be lost, leading to inaccurate determinations of the ratios between free and esterified forms. The second method involves enzymatic assays performed directly on the sample [2], which, however, only determine the concentra- tions of free and total (free + esterified) cholesterol, the concentration of esterified cholesterol being derived from the difference. Since the results may be influenced by various physiological parameters, such as hypertriglyceridemia, and since the measurement of free cholesterol is not very precise, the results should be interpreted carefully.

Recently, new methods based on ${ }^{1} \mathrm{H}$ NMR have been developed, which permit direct measurements on blood plasma samples of their lipoprotein and lipid contents [3-5]. As described, however, these methods depend on the accuracy of the biochemical characterisation of the samples used for calibration and/or calculation of the NMR proton visibility for each lipoprotein. Hence, this calibration needs to be particularly accurate. Nevertheless, in all the publications cited [3-5] the biochemical analysis was carried out using enzymatic kits, where only total cholesterol and cholesteryl ester weights were measured. The methods based on ${ }^{1} \mathrm{H}$ NMR have proved to be very powerful and the results obtained compare favourably with enzymatic kits. Their precision and speed of analysis are probably sufficient for most clinical applications. In their present form, however, they do not provide a better analysis than enzymatic kits and suffer from the need of an external calibration. Therefore, it is apparent that a new method for measuring cholesterol and cholesteryl ester concentrations more precisely than is possible with the enzymatic kits and with less manipulation than required by chromatography is needed.

* Correspondence and reprints.

Received October 29, 1999; accepted November 26, 1999. 
This paper proposes a direct method using ${ }^{13} \mathrm{C}$ NMR and a calibrated internal standard. It has long been recognised that ${ }^{13} \mathrm{C}$ NMR spectroscopy offers a high potential for the quantitative analysis of complex mixtures, due to the wide spectral range and, usually, an absence of interference between peaks. Only recently, however, have instruments of sufficient field strength for biological studies at natural abondance been available. ${ }^{13} \mathrm{C}$ NMR suffers, however, from poor sensitivity due to the low abundance and small magnetic moment of the ${ }^{13} \mathrm{C}$ nucleus. This sensitivity is further diminished if the usual conditions for quantitative NMR inverse-gated decoupling for NOE suppression [6] and a repetition time of 5 to 10 times the longest spin-lattice relaxation time [7-9] - are employed. The use of such acquisition conditions will frequently lead to unacceptably long accumulation times to obtain an acceptable signal/noise ratio for a solute at low concentration. The alternative is to add a paramagnetic relaxation reagent [10], such as triacetylacetonatochromium (III) $\mathrm{Cr}(\mathrm{AcAc})_{3}$, which shortens the relaxation times. However, the invasive addition of such agents may lead to undesirable alteration of the properties of biological samples.

It was shown 20 years ago that the relative concentrations of molecules with similar structures (and similar $\mathrm{T}_{1}$ values) can be determined rapidly by ${ }^{13} \mathrm{C} \mathrm{NMR}$, without added paramagnetic relaxation reagent nor NOE suppression [11]. We have applied this method for the quantification of free and esterified cholesterol in various mixtures, with known amounts of cholesteryl bromide added as an internal concentration reference. Cholesteryl palmitate, a natural cholesteryl ester present in human plasma, was chosen as a model to represent esterified cholesterol. The method was then applied to a mixture of free and esterified cholesterol extracted from a human blood sample and shown to determine accurately their concentrations.

\section{Materials and methods}

\section{Materials}

Cholesterol (5-cholesten-3 $\beta$-ol, purity > $99 \%$ ), cholesteryl bromide ( $3 \beta$-bromo-5-cholestene, purity > $99 \%$ ) and cholesteryl palmitate (5-cholestene-3-palmitate, purity > $91 \%$ ), were purchased from Sigma Chemical Co. (St. Louis, USA) and were used without further purification. Deuterated chloroform $\left(\mathrm{CDCl}_{3}\right.$; purity > $\left.99.8 \%\right)$, obtained from S.D.S. (Peypin, France), was used as the solvent in all the samples. Tetramethylsilane (TMS; purity $99.9 \%$ ), supplied by Aldrich Chemie (Steinheim, Germany), was used as the internal chemical shift reference. Cholesterol and its derivatives were first studied separately. Ten mixtures of cholesterol, cholesteryl bromide and cholesteryl palmitate of concentrations between 5 and $100 \mathrm{mM}$ in $\mathrm{CDCl}_{3}$ were also prepared, by successive dilutions.

A sample of human blood plasma was taken at the Hospital Hôtel-Dieu, Nantes (France). Low density lipoproteins (LDL) were isolated by ultracentrifugation [12]. Lipids were extracted by the Folch method [13]. One aliquot of the extract was submitted to chromatography on silicic acid using microcolumns Waters [1] to separate cholesterol and cholesteryl esters. The samples were then assayed enzymatically using the CHOD-PAD (Boehringer) kit [2]. A second aliquot of the extract was used for NMR analysis after dissolution in $1 \mathrm{ml}$ of $\mathrm{CDCl}_{3}$ and addition of $6.10 \mathrm{mg}$ of cholesteryl bromide.

\section{Methods}

All NMR spectra were recorded at $11.7 \mathrm{~T}$ and $301 \mathrm{~K}$ on a Bruker Avance 500 DRX spectrometer operating at ${ }^{13} \mathrm{C}$ frequency of $125.76 \mathrm{MHz}$, using a $5 \mathrm{~mm}$ dual probe. In general, free induction decays (FIDs) for ${ }^{13} \mathrm{C}$ were accumulated with $64 \mathrm{~K}$ time-domain data points, a spectral width of $25 \mathrm{kHz}$, an acquisition time of $1.30 \mathrm{~s}, 90^{\circ}$ read pulses $(6.3 \mu \mathrm{s})$ and a repetition time of $4.3 \mathrm{sec}$ with continuous, power-gated ${ }^{1} \mathrm{H}$ decoupling (WALTZ-16). The spectra of cholesterol, its derivatives and the mixtures I-X were acquired with 2000 transients, while 32000 transients were used for the plasma extract (corresponding to an experiment length of $11.5 \mathrm{~h}$ ). Spin-lattice relaxation times $\left(T_{1}\right)$ of cholesterol and its derivatives were measured using the inversion-recovery method with power-gated ${ }^{1} \mathrm{H}$ decoupling and a repetition time of $11.3 \mathrm{~s}$. Nuclear Overhauser enhancement factors $(\eta)$ for the different carbon sites were obtained by comparison of the corresponding peak areas $\mathrm{I}_{\text {sat }}$ and $\mathrm{I}_{0}$ obtained with continuous and inverse-gated ${ }^{1} \mathrm{H}$ decoupling, respectively [6], according the relationship $1+\eta=I_{\text {sat }} / I_{0}$.

For data processing, an exponential window function with a line broadening of $2.0 \mathrm{~Hz}$ was applied to the FID prior to Fourier transformation. The phase and baseline of the spectra were corrected manually. Chemical shifts were referenced to TMS. For solutions I-X, peak areas were measured by manual integration. For plasma extracts, they were measured by Lorentzian deconvolution with the Bruker program $\mathrm{X}$-WinNMR 1.1.1. The concentrations of cholesterol and esterified cholesterol were calculated from the area of a given ${ }^{13} \mathrm{C}$ resonance relative to the area of the corresponding resonance for cholesteryl bromide, the reference molecule. These results were then averaged over the different carbons studied.

\section{Results and discussion}

The chemical structure and numbering system for cholesterol and its derivatives are shown in figure 1. As can be seen from the ${ }^{13} \mathrm{C}$ NMR spectra of individual samples of free cholesterol, esterified cholesterol and cholesteryl bromide (Fig. 2), cholesteryl bromide shows a similar spectrum to the metabolites under study but with a small shift in a number of resonances, due to the change in the C-3 substituent. Its similar structure to the molecules under study, combined with its ${ }^{13} \mathrm{C}$ spectrum, determined the choice of cholesteryl bromide as the best reference compound from a range of cholesterol derivatives studied. Peak assignments were made according to the literature $[11,14,15]$. A detailed 


\section{Original articles}

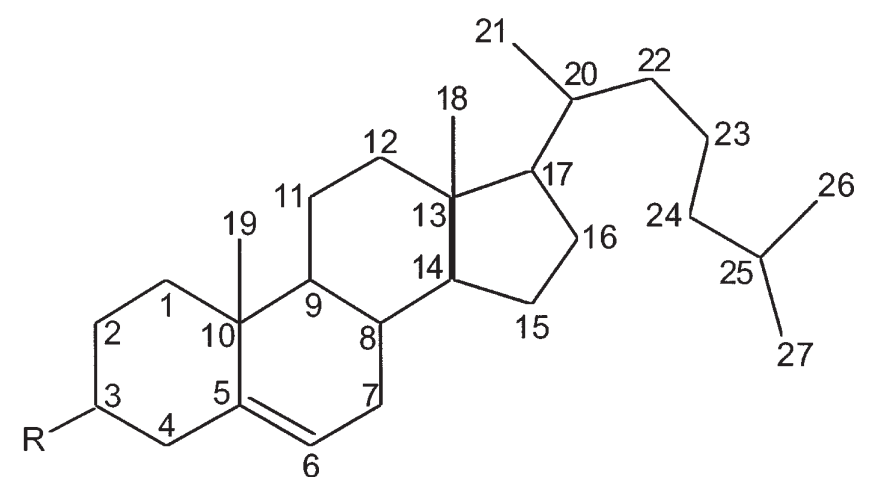

Fig. 1. Structure and numbering system of cholesterol $(\mathrm{R}=\mathrm{OH})$, cholesteryl bromide $(\mathrm{R}=\mathrm{Br})$ and cholesteryl palmitate/esterified cholesterol ( $\mathrm{R}=$ fatty acid).

comparison of the spectra revealed that $\operatorname{six}{ }^{13} \mathrm{C}$ NMR signals (C-1 to C-6) may be used to quantify esterified cholesterol (Fig. 2). Only five ${ }^{13} \mathrm{C}$ signals can be used to determine the free cholesterol concentration since the resonances for $\mathrm{C}-4$ and $\mathrm{C}-13$ overlap (Fig. 2a). Furthermore, the C-2 resonances in free and esterified cholesterol (at 31.7 and $27.8 \mathrm{ppm}$ respectively) are close to several other signals, which may lead to increased error limits for the analysis via this particular carbon.
Figure 3 summarises the spin-lattice relaxation times $T_{1}$ and NOE factors $\eta$ for all carbons of interest for cholesterol, cholesteryl bromide, cholesteryl palmitate and an esterified cholesterol fraction in $\mathrm{CDCl}_{3}$. The data for cholesteryl palmitate and the esterified cholesterol fraction correlate reasonably well, except for the $T_{1}$ of C-6 and the NOEs for C1 and C-3. The chemical shifts of C-3, C-5 and C-6 in cholesterol and esterified cholesterol are similar to those found in intact lipoproteins [16]. Therefore, it can be conducted that cholesteryl palmitate acts as an effective model for esterified cholesterol extracted from plasma.

With these parameters, an estimate of the systematic errors expected for concentration measurements can be made, when a pulse sequence with a short repetition time and ${ }^{1} \mathrm{H}$ decoupling (NOE) are employed. Using a known weight of cholesteryl bromide as internal reference, a correction factor, $f_{\text {corr }}$, for each carbon resonance, can be introduced, by which the measured magnetisation should be multiplied, so as to obtain the expected concentration relative to the reference. This parameter depends on the corresponding NOE and $T_{1}$ values:

$$
f_{\text {corr }}=\frac{\left(1+\eta_{\text {ref }}\right)}{\left(1+\eta_{\text {comp }}\right)}\left(\frac{1-\exp \left(-T_{R} / T_{1, \text { ref }}\right)}{1-\exp \left(-T_{R} / T_{1, \text { comp }}\right)}\right)
$$

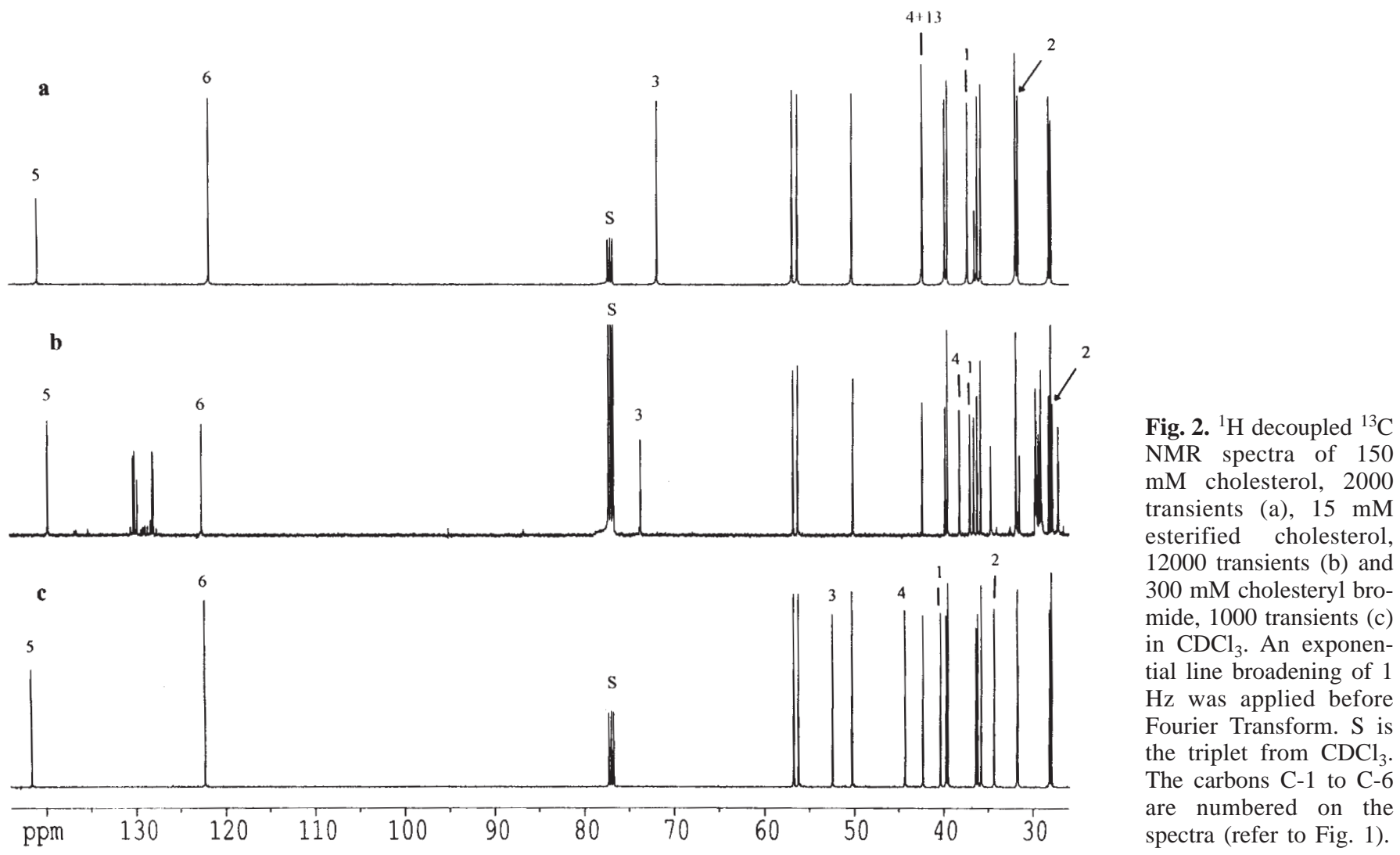




\section{Original articles}

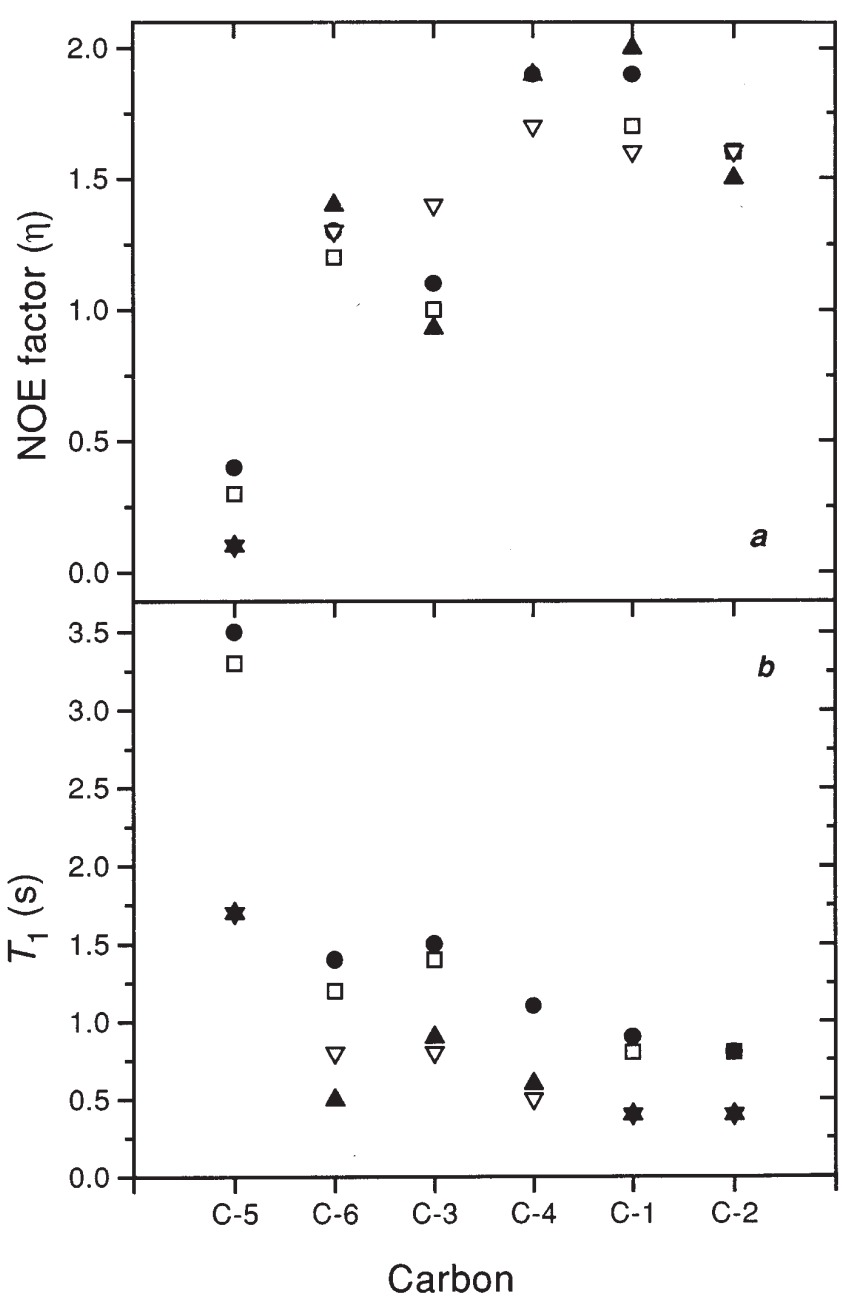

Fig. 3. Nuclear Overhauser enhancement factors $\eta$ (a) and spin-lattice relaxation times $T_{1}$ (b) of C-1 to C-6 of cholesterol ( $\square$ ), cholesteryl bromide $(\mathbf{)})$, cholesteryl palmitate $(\nabla)$ and esterified cholesterol (A). C-4 of cholesterol is not represented, as it overlaps with C-13.

where $\eta_{\text {ref }}, \eta_{\text {comp }}, T_{1, \text { ref }}$ and $T_{1, \text { comp }}$ are the NOE and $T_{1}$ values for the corresponding resonances of the reference compound and the mixture component respectively, and $T_{R}$ is the repetition time of the pulse sequence ( $4.3 \mathrm{sec}$ in this case). Calculated correction factors for the different cholesterol derivatives are shown in figure 4 , and have values between 0.9 and 1.1. Thus, the systematic error for any individual ${ }^{13} \mathrm{C}$ signal used for quantification without using $f_{\text {corr }}$ should not be larger than $10 \%$. Furthermore, by using the statistical average over a number of ${ }^{13} \mathrm{C}$ signals for each molecular species, a mean systematic error of less than $3 \%$ is obtained. This is illustrated in table I, where the NMRderived concentrations calculated without using $f_{\text {corr }}$ for cholesterol and cholesteryl palmitate in samples I to $\mathrm{X}$, are

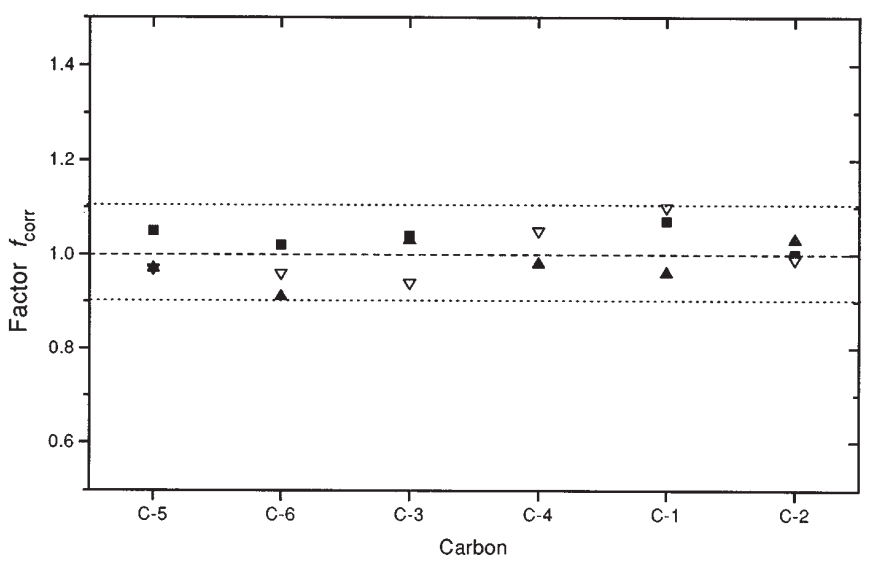

Fig. 4. Correction factors for carbons C-1 to C-6 of cholesterol ( $\square$ ), cholesteryl palmitate $(\nabla)$ and esterified cholesterol $(\boldsymbol{\Delta})$ to the internal reference cholesteryl bromide.

given. It can be seen that there is an excellent correlation between the measured values and the expected concentrations $\left(\mathrm{R}^{2}=0.9998\right.$ for free cholesterol and to $\mathrm{R}^{2}=0.9987$ for esterified cholesterol). This correlation compares very favourably with those usually obtained by ${ }^{1} \mathrm{H}$ NMR for global plasma analysis [3-5]. Thus, the cholesterol analytical method presented here enhances the precision available for the direct NMR analysis on crude samples.

Theoretically, the concentration measurements presented here should be improved by the multiplication of the measured concentration with the correction factors $f_{\text {corr }}$, for each carbon. In practice, however, there was no significant improvement as a result of such data treatment, presumably as the correction factors are all close to 1.0 and may fluctuate a little between the solutions in different concentrations. This implies that the fluctuations of NOEs and $T_{1}$ are sufficiently small and hence that cholesteryl bromide is a good reference.

The ${ }^{13} \mathrm{C}$ NMR spectrum of free and esterified cholesterol in a crude extract of lipids from blood plasma is shown in figure 5 and the derived concentrations are given in table II. The values obtained of $0.33 \mathrm{mg}$ of free cholesterol and $1.29 \mathrm{mg}$ of esterified cholesterol are in good agreement with the results obtained by enzymatic assay after chromatography, 0.34 and $1.30 \mathrm{mg}$, respectively, indicating that the direct determination by NMR provides a valid measurement.

In contrast, measurements made on whole blood plasma samples indicated that at the same temperature, a substantial proportion (about $70 \%$ ) of the esterified cholesterol was "invisible" by NMR (data not shown). A similar, but lower, invisibility was previously noted in ${ }^{1} \mathrm{H}$ NMR measurements $[5,17]$. While this difference indicates that much of the esterified cholesterol is in a slow motion state, further work is required before a firm conclusion can be drawn. 


\section{Original articles}

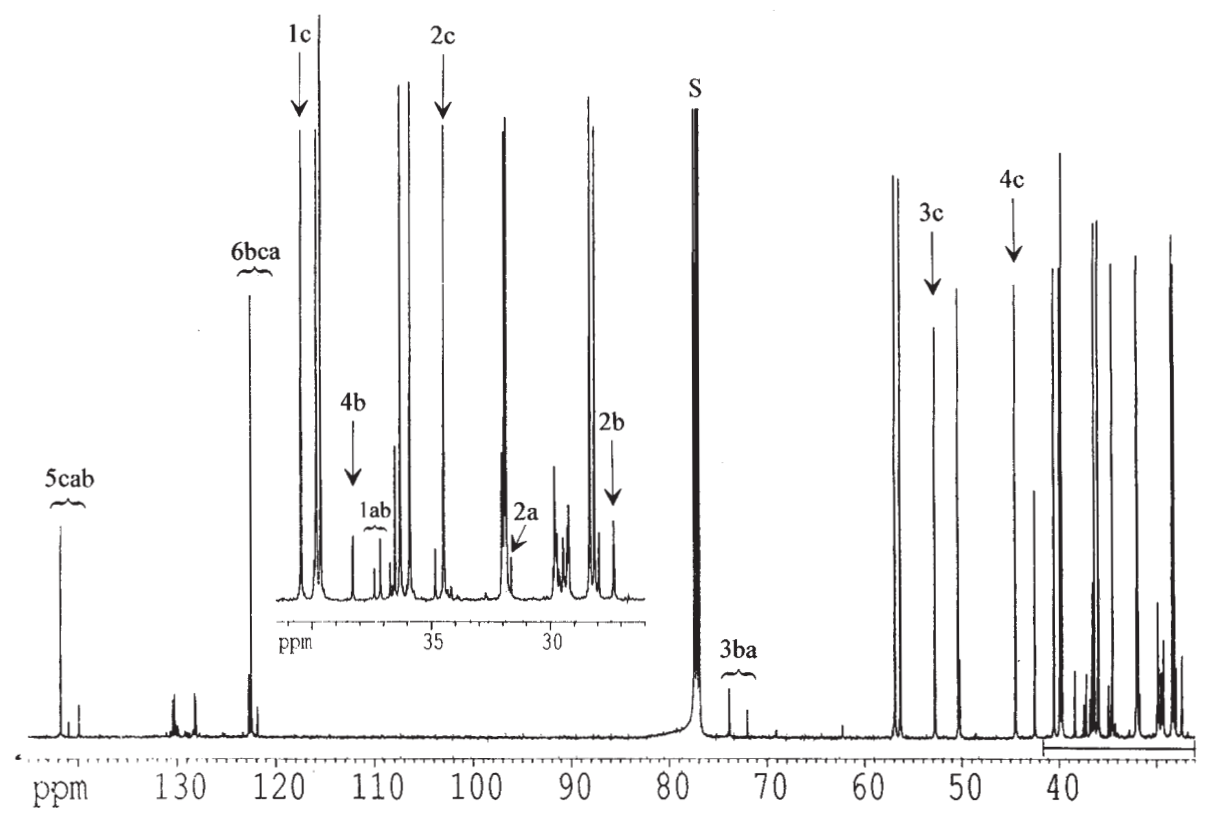

Fig. 5. ${ }^{1} \mathrm{H}$ decoupled ${ }^{13} \mathrm{C}$ NMR spectrum of a crude lipid extract from blood plasma containing cholesterol and esterified cholesterol with added cholesterol bromide $(19.3 \mathrm{mM})$ in $\mathrm{CDCl}_{3} . \mathrm{S}$ is the triplet from $\mathrm{CDCl}_{3}$. The carbons C- 1 to $\mathrm{C}-6$ are numbered on the spectra. The letters $\mathrm{a}, \mathrm{b}$, and $\mathrm{c}$ correspond to cholesterol, esterified cholesterol and cholesteryl bromide, respectively. The insert shows an expansion of the spectrum for the range $26-42 \mathrm{ppm}$.

Table I. NMR-derived concentrations of cholesterol and cholesteryl palmitate in standard mixtures I-X.

\begin{tabular}{|c|c|c|c|c|c|c|c|c|c|c|c|c|c|c|c|c|}
\hline \multirow[t]{2}{*}{ Sample } & \multirow{2}{*}{$\begin{array}{c}\text { Cholestery } \\
\text { Bromide } \\
\text { concent. } \\
(m M)\end{array}$} & \multirow{2}{*}{$C-5$} & \multicolumn{6}{|c|}{ Measured concentration of Cholesterol (mM) } & \multicolumn{8}{|c|}{ Concentration of Cholesteryl palmitate ( $\mathrm{mM}$ ) } \\
\hline & & & $C-6$ & $C-3$ & $C-1$ & Mean $\pm S D$ & $\begin{array}{l}\text { Expected } \\
\text { value }\end{array}$ & Diff & $C-5$ & $C-6$ & $C-3$ & $C-4$ & $C-1$ & Mean & $\begin{array}{c}\text { Expected } \\
\text { value }\end{array}$ & Diff. \\
\hline & & & & & & & & & & & & & & & & -1.6 \\
\hline I & & & 1.0 & 3 & & & & & & 43.4 & & & 41.6 & & & -0.2 \\
\hline III & & 74.0 & & & 71 & & & 1.8 & & 33.8 & & & 30.7 & & & -0.1 \\
\hline IV & & 6 & & 52 & 47.9 & & & 1.8 & & 20.8 & & 1 & 20.1 & & & -0.8 \\
\hline $\mathrm{V}$ & & 4 & & 41 & 38 & & & 2.4 & & 17.1 & & & 17.0 & & & 0.4 \\
\hline VI & & & & 29 & 30 & & & & & 11.9 & & & & & & 0.3 \\
\hline VII & & & & 26 & 25 & & & & & 9.02 & & & & & & -0.4 \\
\hline$\sqrt{1}$ & & & & 22 & 21 & & & & & 7.39 & & & 8.79 & & & -0.03 \\
\hline$X$ & & & & 18 & 14 & & & 1. & & 7.42 & & 6. & 6.66 & & & 0.48 \\
\hline$X$ & 12.1 & 9.81 & 14.0 & 14.7 & 12.1 & $12.7 \pm 2.2$ & 12.2 & 0.5 & 7.63 & 4.84 & 6.64 & 3.06 & 4.81 & $5.40 \pm 1.8$ & 5.27 & 0.13 \\
\hline
\end{tabular}

Samples I to X are mixtures of cholesteryl bromide, cholesteryl palmitate and cholesterol in known concentrations, in $\mathrm{CDCl}_{3}$. Concentrations of cholesterol and cholesteryl palmitate were calculated from the signal integrals of four or five carbon atoms relative to those of cholesteryl bromide as internal reference.

Table II. NMR determination of the concentrations of free and esterified cholesterol extracted from human blood.

\begin{tabular}{|c|c|c|c|c|c|}
\hline & \multicolumn{3}{|c|}{ Peak Areas } & \multicolumn{2}{|c|}{ Concentration ( $\mathrm{mM})$} \\
\hline & $\begin{array}{c}\text { Free cholesterol } \\
\text { cholesterol }\end{array}$ & $\begin{array}{l}\text { Esterified } \\
\text { cholesterol }\end{array}$ & $\begin{array}{l}\text { Cholesteryl } \\
\text { bromide }\end{array}$ & $\begin{array}{c}\text { Free } \\
\text { cholesterol }\end{array}$ & $\begin{array}{l}\text { Esterified } \\
\text { cholesterol }\end{array}$ \\
\hline $\mathrm{C}-5$ & 1.62 & 3.98 & 21.6 & 1.45 & 3.56 \\
\hline C- 6 & 3.55 & 9.36 & 51.5 & 1.33 & 3.51 \\
\hline $\mathrm{C}-3$ & 3.43 & 7.39 & 51.5 & 1.29 & 2.77 \\
\hline C-4 & & 8.91 & 55.8 & & 3.08 \\
\hline $\mathrm{C}-1$ & 4.09 & 8.84 & 56.2 & 1.40 & 3.03 \\
\hline $\mathrm{C}-2$ & 4.18 & & 61.2 & 1.32 & \\
\hline Mean \pm SD & & & & $1.36 \pm 0.07$ & $3.20 \pm 0.30$ \\
\hline
\end{tabular}

The concentration of cholesteryl bromide was $19.3 \mathrm{mM}$. The mean concentrations of free and esterified cholesterol correspond to initial weights of 0.33 \pm 0.02 and $1.29 \pm 0.12 \mathrm{mg}$ respectively, which is in good agreement with the results obtained by another method $(0.34 \pm 0.01$ and $1.30 \pm 0.03 \mathrm{mg}$ respectively). 
In summary, a direct and accurate method to quantify free and esterified cholesterol in a mixture by ${ }^{13} \mathrm{C}$ NMR has been described, with the nuclear Overhauser enhancement and the use of a short recycle time. This is achieved by the use of an adequate internal reference and allows precise results in diluted samples to be obtained during a reasonable experiment length. While unlikely to replace "routine" biochemical screening methods, this approach could be useful when a precise measurement of cholesterol and cholesteryl ester concentrations is needed or if the validity of enzymatic measurements requires checking. It necessitates less manipulations than measurements requiring chromatography separation. A unique advantage of this method is that it could also be used for the simultaneous measurement of the ${ }^{13} \mathrm{C}$ enrichments of cholesterol and cholesteryl ester in isotopic tracer studies, if this enrichment is sufficiently different from ${ }^{13} \mathrm{C}$ natural abundance. Thus, for example, the turnover of cholesterol and cholesteryl esters could be determined directly on human subjects, as could the measurement of the relative contributions to the plasma pools of dietary cholesterol and cholesterol synthesized de novo.

\section{Acknowledgements}

R. W. and the L.A.I.E.M gratefully acknowledge a grant of Research Associate from the C.N.R.S. The authors wish to thank Pr Jacques de Certaines and Dr Geneviève Leray for sending pre-prints, Prs Serge Akoka and Maryvonne L. Martin for carefully reading the manuscript and for stimulating discussions, Michel Trierweiler and Françoise Mabon for assistance with the NMR spectrometer.

\section{References}

1. Magot, T.; Malmendier, C.; Ouguerran, K.; Lontie, J.-F.; Lutton, C. Clin. Chim. Acta 1991, 196, 59-68.
2. Ouguerram, K.; Magot, T.; Lutton, C. Metabolism 1996, 45, 4-11.

3. Ala-Korpela, M.; Korhonen, A.; Keisala, J.; Hörkkö, S.; Korpi, P.; Ingman, L. P.; Jokisaari, J.; Savolainen, M. J.; Kesäniemi, Y. A. J. Lipid Res. 1994, 35, 2292-2304.

4. Ala-Korpela, M.; Hiltunen, Y.; Bell, J. D. NMR Biomed. 1995, 8, 235-244.

5. Serrai, H.; Nadal, L.; Leray, G.; Delplanque, B.; de Certaines, J. D. NMR Biomed. 1998, 11, 273-280.

6. Freeman, R.; Hill, H. D. W.; Kaptein, R. J. Magn. Reson. 1972, 7, 327-329.

7. Canet, D. J. Magn. Reson. 1976, 23, 361-364.

8. Harris, R. K.; Newman, R. H. J. Magn. Reson. 1976, 24, 449456.

9. Opella, S. J.; Nelson, D. J.; Jardetzky, O. J. Chem. Phys. 1976, 64, 2533-2535.

10. Shoolery, J. N. Prog. NMR spectrosc. 1977, 11, 79-93.

11. Mareci, T. H.; Nasfay Scott, K. Analyt. Chem. 1977, 49, 21302136.

12. Poupon, R. E.; Ouguerram, K.; Chrétien, Y.; Verneau, C.; Eschwège, E.; Magot, T.; Poupon, R. Hepatology 1993, 17, 577-582.

13. Folch, N.; Lees, M.; Stahley, G. H. J. Biol. Chem. 1957, 226, 497-507.

14. Reich, H. J.; Jautelat, M.; Messe, M. T.; Weigert, F. J.; Roberts, J. D. J. Amer. Chem. Soc. 1969, 91, 7445-7454.

15. Breitmaier, E.; Voelter, W. Carbon-13 NMR spectroscopy; VCH ed.: New York, 1987.

16. Hamilton, J. A.; Morrisett, J. D. In Methods in enzymology; Academic Press Inc., 1986; Vol. 128, pp. 473-515.

17. Miller, N. E.; Lewis, B. Lipoproteins, Atherosclerosis and coronary heart disease; Miller, N. E., and Lewis, B., Ed., Elsevier: North Holland, 1981. 